\title{
CONCILIACIÓN PREJUDICIAL EN MATERIA CIVIL Y FAMILIAR, COMO REQUISITO DE PROCEDIBILIDAD PARA ACCEDER A UN PROCESO JURISDICCIONAL
}

\author{
THE PREJUDICIAL SETTLEMENT IN CIVIL AND FAMILY MATTERS, AS A \\ PROCEDURE REQUIREMENT TO ACCESS A JURISDICTIONAL PROCESS
}

\author{
Adriana de-Jesús Rodríguez-Ramos ${ }^{1} *$ (iD). \\ 1. Escuela Judicial del Estado de Tabasco, México. adyrodguez8@hotmail.com \\ * Autor de correspondencia: Adriana de-Jesús Rodríguez-Ramos, correo electrónico: adyrodguez8@hotmail.com
}

\section{RESUMEN}

La predisposición de las personas a mantener una mentalidad litigiosa y la falta de difusión de los diversos procedimientos que existen para resolver conflictos, ha ocasionado que las partes opten por solucionar sus desacuerdos en la vía tradicional, es decir, a través del proceso jurisdiccional. Sin embargo, para nadie es desconocido la situación crítica que prevalece en el interior de los tribunales de administración de justicia. El incremento del índice en la promoción de demandas que diariamente son ingresadas por los particulares, con la finalidad de resolver sus desacuerdos legales en el ámbito civil y familiar, ha ocasionado un impacto negativo en la resolución de conflictos por la vía jurisdiccional. Situación que se hace notoria en la dilación y rezago de los procesos que se instan con la de finalidad de obtener una justicia pronta y expedita. Ante tal colapso, el tiempo y la necesidad han obligado a buscar diversas opciones o alternativas que pongan fin a conflictos de esta índole, de una manera eficaz, efectiva, simple rápida y económica. El presente artículo se basa en el análisis de la necesidad de materializar el mandato constitucional para acceder a una justicia alternativa a través de la implementación de una instancia prejudicial obligatoria de conciliación en la materia civil y familiar. Procedimiento previo al proceso cuyo objetivo será brindar una auténtica posibilidad de solución de los conflictos de índole civil y familiar, así como abreviar los plazos de resolución de los mismos, el cual deberá ser presidido por funcionarios conciliadores expertos.

Palabras clave: acceso a la justicia; conciliación prejudicial; justicia alternativa; proceso jurisdiccional.

Cómo citar:

Rodríguez-Ramos, Adriana de-Jesús. (2021). Conciliación prejudicial en materia civil y familiar, como requisito de procedibilidad para acceder a un proceso jurisdiccional. Revista de Investigaciones Universidad del Quindio, 33(S2), 191-197. https://doi.org/10.33975/riuq. vol33nS2.631 


\begin{abstract}
The predisposition of people to maintain a litigious mentality and the lack of dissemination of the various procedures that exist to resolve conflicts, has caused the parties to choose to resolve their disagreements in the traditional way, that is, through the jurisdictional process. However, the critical situation that prevails within the courts for the administration of justice is unknown to anyone. The increase in the index in the promotion of lawsuits that are filed daily by individuals, in order to resolve their legal disagreements in the civil and family sphere, has had a negative impact on the resolution of conflicts through jurisdictional channels. Situation that becomes notorious in the delay and delay of the processes that are urged in order to obtain a prompt and expeditious justice. Faced with such a collapse, time and necessity have forced us to look for various options or alternatives that put an end to conflicts of this nature, in an efficient, effective, simple, fast and economic way. This article is based on the analysis of the need to materialize the constitutional mandate to access an alternative justice through the implementation of a mandatory pretrial conciliation instance in civil and family matters. Procedure prior to the process, the objective of which will be to provide a genuine possibility of resolving conflicts of a civil and family nature, as well as shortening the deadlines for their resolution, which must be presided over by expert conciliatory officials.
\end{abstract}

Keywords: alternative dispute resolution mechanisms; access to the justice; alternative justice; prejudicial conciliation; jurisdictional process.

\title{
INTRODUCCIÓN
}

El 18 de junio de 2008 se implementa una reforma transcendental al artículo 17 de la Constitución Política de los Estados Unidos Mexicanos: el reconocimiento de los mecanismos alternativos de resolución de conflictos como un derecho humano.

Tal norma constitucional, en su párrafo cuarto, literalmente señala: "Las leyes preverán mecanismos alternativos de solución de controversias" (CPEUM, 2011, Artículo 17, párrafo cuart0). Innovación constitucional que contempla la obligatoriedad de la justicia alternativa para todas las áreas del derecho, sin distinción alguna.

Ahora bien, no se desconoce que dentro del propio proceso jurisdiccional siempre ha existido como una fase obligatoria del procedimiento la etapa conciliatoria. Periodo cuyo objetivo consiste en lograr un avenimiento entre las partes interesadas, el cual incluso se puede intentar en cualquier etapa del juicio hasta antes de que se dicte sentencia (Código Civil del estado de Tabasco, 2020, Artículo 3), a través de la celebración de un convenio que ponga fin a la controversia planteada. No obstante, con el objetivo garantizar un verdadero acceso a la justicia mediante los mecanismos alternativos de resolución de conflictos, se hace menester hacer uso de estos procedimientos o vías, de manera previa a un proceso judicial.

Existe un gran número de conflictos civiles y familiares que son sometidos inicialmente a la decisión de las y los jueces para su resolución. Empero, en la etapa conciliatoria 4 de cada 10 casos, han logrado ser resueltos a través de la conciliación, es decir, las propias personas integrantes del conflicto se dan la oportunidad de acceder a la justicia alternativa. 
Ante esta situación, al existir un gran índice de casos que pueden ser resueltos por las propias partes, con ayuda de un tercero imparcial al conflicto, resulta funcional hacer visible la materialización del contenido del artículo 17 constitucional al contemplar en los códigos civiles y familiares la conciliación prejudicial como un mecanismo que da solución a una necesidad de justicia.

\section{METODOLOGÍA}

En el presente artículo se analiza la importancia de la conciliación prejudicial en el ámbito civil y familiar. El método empleado es el deductivo, porque parte de lo general (la conciliación prejudicial como requisito de procedibilidad), aplicándose a casos concretos de índole civil y familiar para analizar el acceso a la administración de justicia, al debido proceso y la justicia pronta y expedita.

A la vez se utiliza la índole explicativa, dado que se enumeran las ventajas de la institución de la conciliación prejudicial, siendo una de estas la descongestión judicial. El enfoque de la investigación es cualitativo, por tener tanto elementos cualitativos como cuantitativos. Cualitativos, porque analiza heurísticamente la institución jurídica de la conciliación prejudicial con el objetivo de demostrar el impacto positivo en la descongestión judicial y en el debido proceso.

Se emplea el método exegético que permite analizar e interpretar la normativa referida a la justicia alternativa en materia civil y familiar. Asimismo, se aborda de forma local un acercamiento a las estadísticas para demostrar que los mecanismos alternativos son un mecanismo idóneo, eficaz y efectivo para garantizar derechos fundamentales, tales como el acceso a la administración de justicia. Para ilustrar lo anterior se citará, como ejemplo, la experiencia judicial en las que se propone cómo la conciliación como mecanismo alternativo, coadyuva a hacer efectivo el acceso a la administración de justicia, de manera pronta, expedita e imparcial.

\section{RESULTADOS}

\section{El proceso jurisdiccional como forma de solución a los conflictos.}

A través del tiempo, se han desarrollado diversas formas de solución de conflictos. Entre estas se encuentran la autotutela, la autocomposición y la heterocomposición (Alcalá-Zamora \& Castillo, 1947).

Empero, aun cuando existen diversos métodos de resolver un conflicto, se ha considerado al proceso, como la vía más idónea de resolución. Tan es así, que cuando los particulares se encuentran involucrados en un conflicto o controversia de carácter legal, no dudan en elegir como primera y a veces como única opción, un mecanismo heterocompositivo, como es la vía judicial o la instancia jurisdiccional. Esto con el fin de conseguir la solución a su conflicto de intereses. Resolución que, en la mayoría de los casos, engendra inconformidad en las partes involucradas, al no obtener la solución que esperaban afín a sus intereses.

Situación que obliga a las partes del conflicto a recurrir a otras instancias, con el fin de revocar o modificar la determinación asumida por la o el juzgador de primer grado. Prologándose de esta manera la resolución definitiva de la controversia planteada, que impide la solución de la litis de una manera rápida, eficaz y eficiente. 
Cabe hacer notar, que esta manera "tradicional" de resolución de conflictos, hace palpable la desconfianza que se tiene en uno mismo, al poner en tela de juicio las aptitudes y capacidades con la que cada persona cuenta para manejar el conflicto e incluso, para elegir la mejor solución que se acomode a sus intereses.

Esto se afirma, debido a que cuando las partes deciden resolver sus intereses opuestos a través de un procedimiento judicial, la solución de su conflicto la delegan a un tercero ajeno a la controversia. Quien impondrá a las partes conflictuadas una solución que éstas esperan sea apegada a la verdad, certera, justa y oportuna.

En ese sentido, tradicionalmente se visualizó que el Estado ejercía la función jurisdiccional en forma exclusiva (Rojas, 2002). Por tanto, los justiciables se sometieron por mucho tiempo a la idea de que era el Estado a través de sus órganos jurisdiccionales quien debía de emitir la solución válida y legal a las controversias o desacuerdos que se suscitaran entre los gobernados.

Sin embargo, ante la problemática del colapso por la que atraviesan los tribunales de justicia, emergida de su morosidad ostensible, lo cual no solo es atribuible a los funcionarios judiciales, sino también a la elevada conflictividad entre los gobernados y la nula difusión de procedimientos diversos al judicial, que otorguen a las partes, la oportunidad de resolver la controversia de manera participativa (mecanismo autocompositivo) a través de soluciones envestidas de equidad, justicia, seguridad jurídica y eficacia.

Surge la imperiosa necesidad de obtener los objetivos y finalidades del Estado, enfrentado a una problemática de congestión judicial implementándose los denominados mecanismos alternativos de solución de conflictos. Procedimientos que brindan un catálogo de opciones a los particulares, para resolver su controversia o conflicto de manera pacífica, sin necesidad de someterse a una contienda judicial que en muchos casos trae desgaste económico, emocional, psicológico e incluso demanda tiempo, ante el desahogo de audiencias y/o comparecencias.

\section{La conciliación prejudicial.}

La expresión conciliación proviene del latín conciliatio y en su formulación verbal conciliare. En su acepción general significa acción y efecto de conciliar, conformidad o semejanza de una cosa con otra (Casares, 2012).

Otra definición de conciliación, es concebida como "el acuerdo o aveniencia de partes que, mediante renuncia, allanamiento o transacción, hacen innecesario el litigio pendiente o evitan el litigio eventual", (Couture, 1960).

Una tercera noción de conciliación, podría ser "la comparecencia necesaria o facultativa de las partes en un conflicto de intereses, ante un tercero (sin que la doctrina limite esta concepción a una autoridad), para que en su presencia traten de solucionar el conflicto que las separa”, (Nuñez Ojeda, 2009).

Por su parte, el artículo 3, fracción II, de la Ley de Acceso a la Justicia Alternativa para el Estado de Tabasco, define la conciliación como: "Procedimiento a través del cual un especialista propone soluciones a las partes involucradas en un conflicto jurídico, con la finalidad de facilitar el diálogo y la búsqueda de acuerdos voluntarios en común”. 
De las anteriores definiciones, podemos concluir que conciliar, supone avenimiento entre intereses contrapuestos. Es establecer armonía entre dos o más personas con posiciones contrarias.

De este modo, no es desconocido para la mayoría de las personas que han estado vinculadas a un proceso jurisdiccional, que dentro del mismo procedimiento existe una etapa conciliatoria como una fase procesal, en el que se concede a las partes del juicio un espacio de oportunidad para avenir la controversia legal sometida al conocimiento del juzgador.

Pero en la práctica lo que se observa es que no se le da la importancia que se debe a esta etapa, ya que tanto las partes como los mismos jueces, simplemente lo toman como una etapa procesal, que deben agotar para llegar a la etapa final del proceso, es decir, la sentencia.

En la práctica se estila que, dentro de la audiencia conciliatoria, el juez o conciliador, en la mayoría de las ocasiones se limitan a conceder el uso de la voz a las partes para que manifiesten si tienen ánimo de conciliar. Generalmente las partes no cuentan con el ánimo conciliatorio y lo manifiestan, razón por la cual el juez debería intentar proponer fórmulas de arreglo y persuadir a las partes para que solucionen el conflicto en esa etapa, pero caso contrario lo que se presenta es que el juez da por terminada esa etapa procesal y continúa con la secuela procesal.

Siendo esta, una de las razones por la cual se fracasa en parte la mayoría de conciliaciones judiciales, por la falta de diligencia, esmero e importancia que el juez le debe aplicar a etapa procesal.

No obstante, la situación antes descrita. También existe un gran número de casos, en los que las propias partes, se dan la oportunidad de encontrar una solución pronta, eficaz y efectiva a su conflicto, a través del uso de procedimientos alternos al judicial como es la conciliación.

Según el libro de conciliaciones que se lleva en cada juzgado del Estado de Tabasco, específicamente en los juzgados familiares, de manera mensual se refleja una cifra constante entre 15 a 20 acuerdos conciliatorios celebrados dentro del procedimiento. Es decir, mensualmente se están archivando definitivamente de 15 a 20 juicios en trámite.

Lo anterior hace suponer, que si el procedimiento de conciliación fuera extra procesal, o previo al proceso, se estaría evitando el ejercicio del aparato judicial de 15 a 20 controversias familiares. Generando con ello, una disminución de las demandas recepcionadas al interior de los juzgados civiles y familiares, debido a la innecesaria promoción de juicios del orden civil y familiar, por haber sido resueltos, a través de la justicia alternativa.

En esa perspectiva, ante las situaciones que prevalecen ante el uso de la justicia participativa (mecanismos alternativos), debe difundirse y enfocarse el acuerdo surgido del proceso de conciliación, como la primera vía para resolver conflictos familiares y civiles.

Esta nueva forma encuentra su justificación en que los sistemas de justicia tradicionales se han sobrecargado de conflictos, de tal manera que provoca demoras y altos costos para los justiciables.

Son muchos los factores que justifican recurrir a la conciliación (Gerónimo et al., 2021) para dirimir también los conflictos civiles y familiares. En efecto, la búsqueda de soluciones alternativas y diferentes a la justicia tradicional es y sigue siendo muy frecuente. 
Sehapermitido recurrir a mecanismos alternativos para solucionar los desacuerdos, comola conciliación, porque son más simples, más rápidos, más efectivos, en muchos casos más baratos, directos, e incluso más transparentes que la justicia formal y tradicional. Esto debido a la desconfianza generada por los órganos de justicia oficiales, en muchas ocasiones con un alto índice de congestionamiento de demandas.

En ese orden de ideas, además de difundir y promocionar el uso de un mecanismo alternativo, lo que se pretende a través de la regulación de la obligatoriedad de la conciliación prejudicial, es que los conflictos no conciliables o más difíciles del orden civil y familiar, son los que tengan que decidirse o resolverse a través de la forma heterocompositiva tradicional, es decir, la vía judicial.

\section{Beneficios de la conciliación en el ámbito civil y familiar.}

En un sinnúmero de casos, "la verdad legal" dista mucho de lo que las partes pueden considerar una solución justa al conflicto.

La onerosidad de lo que significa llevar un proceso por la vía judicial, la demora con la cual normalmente son resueltos los casos por los tribunales jurisdiccionales, derivados del alto índice de demandas y la impredecibilidad del fallo. Crea una incertidumbre jurídica en las partes que demandan la solución a un conflicto.

Sin embargo, el precepto constitucional (artículo 17), sin duda, abre el paragua de acceso a la justicia ya que, si se ve de manera positiva la conciliación prejudicial obligatoria, es la oportunidad de la solución a los conflictos de índole civil y familiar en una vía más corta y menos costosa, mediante acuerdos voluntarios que sean conforme a los intereses de las partes.

La instancia conciliatoria prejudicial obligatoria para acudir a la jurisdicción, no entorpece o violenta el derecho de acceso a la justicia, porque, la participación en un procedimiento de conciliación bien se puede agotar en el período que tiene el titular del derecho para interrumpir la prescripción. De tal suerte que, si no llegan a acuerdos en la instancia de conciliación, tienen expedita la vía para la jurisdicción. Así pues, la instancia prejudicial permite a los dueños del conflicto encontrar soluciones más ágiles y económicas y, consecuentemente, mayor eficacia sin merma de su aceptabilidad social.

Con la conciliación previa a la jurisdicción, se pretende crear una instancia ágil y menor costo económico para las personas, así como cumplir con los principios de justicia pronta y expedita consagrados en el artículo 17 Constitucional varias veces aludido.

Luego, la conciliación prejudicial obligatoria, debe visualizarse como una alternativa viable para solucionar los problemas de los particulares de una manera rápida, eficaz y con el menor daño en las relaciones interpersonales.

Mecanismo que, además, coadyuva en la descongestión de la función judicial, colaborando con el órgano jurisdiccional en su función y quehacer diario. En este orden la conciliación prejudicial obligatoria debe impulsarse y difundirse con el fin de que los gobernados conozcan otras alternativas de solución a su conflicto, con el objetivo de que hagan uso de esta herramienta verás, útil y regulada jurídicamente, sin que quede como letra muerta. 


\section{DISCUSIÓN}

La idea planteada en este trabajo, es difundir e implementar las herramientas que la ley otorga para la solución de los conflictos y que, por cuestiones personales, intrínsecas de las personas, o ausencia de conocimiento no se aprovechan.

No existe impedimento legal alguno para que el Código de Procedimientos Civiles regule de manera taxativa ciertos requisitos de procedibilidad de la acción en determinados juicios (civiles y familiares), lo que en nada se contrapone con el principio general de legalidad ni con el de voluntariedad que caracteriza a los Mecanismos Alternativos de Solución de Conflictos.

Dado que lo que se pretende es que las partes involucradas en un conflicto, se sometan de manera previa al proceso, a una vía distinta de resolución de conflicto en la que tendrán la oportunidad de participar o crear su propia solución, aplicando sus propias capacidades, aptitudes y talentos, que le permita innovar y fomentar una justicia participativa, con la que se evite que los conflictos no difíciles tengan que resolverse dentro de un proceso judicial.

Aunado a ello, que este procedimiento previo se traducirá en una herramienta jurídica les va a brindar, no solo una seguridad jurídica sino una manera rápida, eficaz y útil para la solución de sus conflictos.

\section{REFERENCIAS}

1. Alcalá Zamora \& Castillo Niceto. (1947), Proceso, Autocomposición y Autodefensa. Contribución al estudio de los fines del proceso. México: Imprenta Universitaria.

2. Casares, J. (2012). Diccionario ideológico de la lengua española. Barcelona: Casa del Libro.

3. Couture, E. (1960). Vocabulario jurídico. Sexta impresión. Argentina: Ediciones de Palma.

4. Diario Oficial de la Federación. (2011). Constitución Política de los Estados Unidos Mexicanos.

5. Enrique Rojas, Miguel. Teoría del proceso. Universidad externada de Colombia, $1^{a}$ edición Bogotá 2002, pág. 46

6. Gerónimo González, Paulino, Silva Hernández, Francisca \& Martínez Prats, Germán. (2021). Mecanismos alternativos en la solución de conflictos para la construcción de una cultura de paz. Revista Ciencias de la Documentación, 7(1), 15-23.

7. Núñez Ojeda, Raúl, Negociación, mediación y conciliación, como métodos alternativos de solución de controversias, Bogotá, Leyer (2009).

8. Periódico Oficial del Estado. (2020). Código Civil del estado de Tabasco.

9. Periódico Oficial del Estado. (2012). Ley de Acceso a la Justicia Alternativa para el Estado de Tabasco.

10. Rojas Franco, Enrique. (2002). Los límites de la jurisdicción constitucional en relación con la jurisdicción contenciosa administrativa en Costa Rica. IIJ-UNAM: México. 